\title{
DIPOLE-MODE-FREE AND KICK-FREE 2-CELL CAVITY FOR THE SC ERL INJECTOR
}

\author{
V. Shemelin", H. Padamsee, S. Belomestnykh, R. L. Geng, M. Liepe \\ Laboratory for Elementary-Particle Physics, Cornell University, Ithaca, NY 14853
}

\begin{abstract}
For the ERL injector, superconducting cavities are needed to deliver to the beam a $100 \mathrm{kWCW}$ RF power. With a beam current of $100 \ldots 33 \mathrm{~mA}$, gap voltage of $1 \ldots 3$ $\mathrm{MV}$, the coupler must have an external $Q$-factor in the range of $4.6 \times 10^{4} \ldots 4.1 \times 10^{5}$. The cavity shape and coupler design presented provide the possibility of working in the range of parameters without substantial transverse kick to the beam and HOM-losses in the system. In order to preserve field flatness while the dipole mode is driven out, the 2-cell cavity has a protruding iris between the cell and the larger beam pipe. A twin-coaxial coupler has high coupling but low kick because of its symmetry. Calculation and optimization of the coupler-cavity system are performed with a 2D SLANS and 3D Microwave Studioß codes.
\end{abstract}

\section{INTRODUCTION}

A multi-GeV Energy Recovery Linac (ERL), proposed by Cornell University in collaboration with Jefferson Lab, is a low emittance, high average beam current $\mathrm{CW}$ accelerator for X-ray science [1]. An injector and a main linac of the ERL are based on the superconducting RF technology.

One of driving ideas behind this machine is to create a low emittance beam using a high-brightness photoemission electron gun and then to preserve the emittance while the beam is accelerated in the injector and in the main linac. The goal is to have the beam with the normalized emittance of $2 \mathrm{~mm} \cdot \mathrm{mrad}$ in undulators. An extension of existing technology in several directions is required to achieve this ultimate goal. Development of a $100 \mathrm{MeV}, 100 \mathrm{~mA}$ average current ERL prototype is in progress at Cornell University [2].

One of the possible sources of emittance dilution is a kick caused by non-zero on-axis transverse electromagnetic fields of fundamental power couplers in superconducting cavities. This effect is especially strong in the injector cavities, where a high average RF power per cavity must be coupled to a vulnerable low-energy beam. The requirements here are far more demanding than in any existing system. Our design goal is to allow a maximum emittance growth of no more than $10 \%$ total for five injector cavities out of the initial emittance of 1 $\mathrm{mm} \cdot \operatorname{mrad}[3]$.

The five injector cavities are superconducting 2-cell niobium structures. They provide a total of $500 \mathrm{~kW}$ of RF power to the beam. Consequently, the permitted beam

*vs65@cornell.edu current depends on the injector energy and varies from $100 \mathrm{~mA}$ at $5 \mathrm{MeV}$ to $33 \mathrm{~mA}$ at $15 \mathrm{MeV}$. The injector cavity coupler has to deliver to the beam $P_{\text {beam }}=100 \mathrm{~kW}$ of RF power and provide matching conditions for a cavity gap voltage of $V=1$ through $3 \mathrm{MV}$. In accordance with the formula

$$
Q_{\text {ext }}=\frac{V^{2}}{R / Q \cdot P_{\text {beam }}},
$$

where $R / Q$ is the characteristic impedance of the cavity, the coupler must be adjustable with the external $Q$ factor overlapping the range from $4.6 \times 10^{4}$ to $4.1 \times 10^{5}$. Such small values of $Q_{\text {ext }}$ may demand a deep insertion of the antenna into the beam pipe that adds to the problem of the kick.

There are several possibilities to completely or partially suppress the transverse kick from the fundamental RF power coupler and associated with it emittance growth. They were analyzed in detail in [4]. We settled on the twin-coaxial coupler as a more practical option.

Another source of the emittance dilution is an interaction of the beam with high $Q$ transverse higherorder modes (HOMs). Especially dangerous are lowest dipole modes whose frequencies can be below cut-off frequency of the beam pipes. However, it is possible to find a cavity shape so that the frequency of the lowest dipole mode is high enough to propagate into the beam pipe. In this case its impedance becomes small as well as its impact on the beam. Examples of such shapes are single-cell superconducting cavities developed at Cornell [5] and at KEK [6].

In this paper we present the 2-cell superconducting cavity shape with propagating dipole modes in the presence of the twin-coaxial coupler. 2D codes SLANS and SLANS2 [7] were used to optimize the cavity shape. The 3D MWS 4.0 code was used to verify the obtained results, to optimize the coupler and to study the dipole modes in the presence of the coupler. More details about the optimization can be found in [8].

\section{CELL SHAPE OPTIMIZATION}

Having the same frequency as in the TESLA project [9] and planning to use TESLA-like multi-cell cavities in the main linac of the ERL, we first chose the shape of the 2cell cavity also TESLA-like (Fig. 1a). However, it turned out that this geometry has a trapped dipole mode. In order to allow this mode to propagate into the beam pipe we decided to use the KEK approach by enlarging one of the beam pipes. We chose the inner radius of one of the beam pipes and the radius of the iris equal to these of TESLA. 
Scaling of the KEK single-cell dipole-mode-free cavity gave us bigger inner radii. A decrease of the inner iris radius increases the frequency of the dipole mode significantly, so we stayed at the TESLA value for the inner iris radius. The larger beam pipe, right-hand one on the Figs. $1 \mathrm{~b}$ and $1 \mathrm{c}$, serves for propagating the dipole mode out from the cavity. The right iris (Fig. 1c) secures identity of fields in both cells but does not preclude the coupling of the dipole mode with the beam pipe.

We analyzed the influence of different cavity dimensions on the TE11-mode frequency, on the maximal values of the surface fields, electric and magnetic, and on the value of $R / Q$ of the fundamental mode.
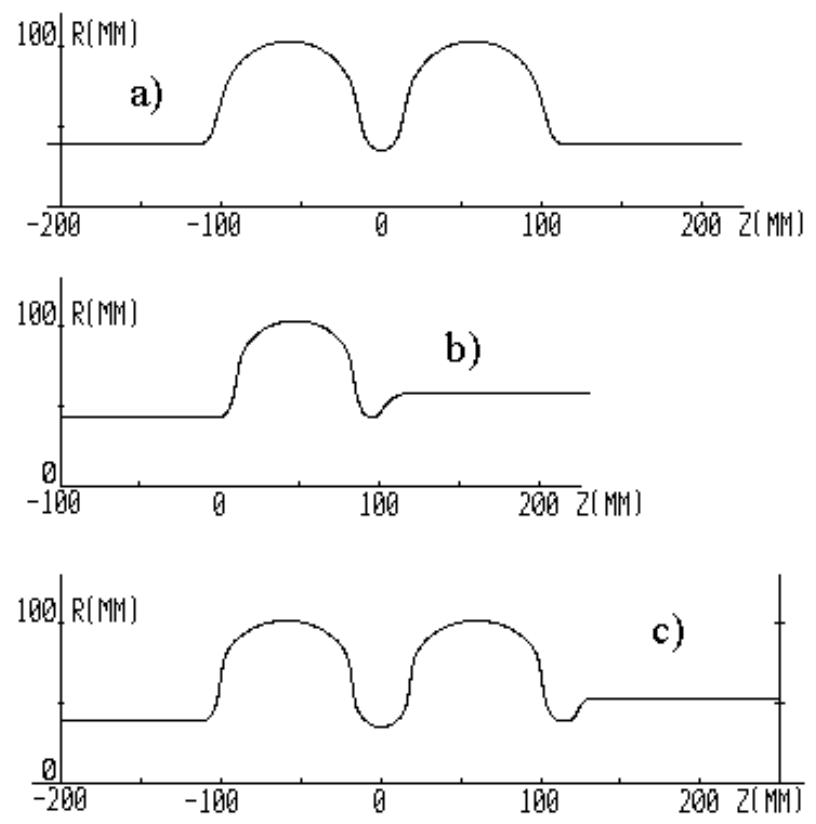

Fig. 1. a) Geometry with a trapped dipole mode (TESLAlike), b) KEKB geometry with a propagating dipole mode, c) Optimized geometry for the ERL injector with a propagating dipole mode.

The frequency of the dipole (TE11-like) mode was kept at least $10 \mathrm{MHz}$ higher than the cut-off frequency of the beam pipe. The right iris has the same inner radius as the left pipe. The bigger right-hand beam pipe radius causes negligible asymmetry of the fundamental mode

Even having strong restrictions upon the frequency of the dipole mode, we have found main cavity parameters being not much different from those of the TESLA cavity. This can be explained by an additional freedom: we have no limitations to the cell length. As one can see (Fig. 1), the injector cavity has a thicker iris than in TESLA. This is connected with the quest for higher frequency of the dipole mode. The cell-to-cell coupling is weaker $(0.7 \%)$ but still sufficient for 2 cells.

The simulation code MultiPac [10] is used to check multipacting characteristic of the optimized cavity. This cavity shape is free of multipacting according to the criteria of this code. Though the resonant motion of electrons can take place at peak surface electric fields of
$30-40 \mathrm{MV} / \mathrm{m}$, the impact energy is about only $26 \mathrm{eV}$, which is too low to warrant electron multipacting. For the original TESLA shape, resonant trajectories also exist with an average impact energy of $32 \mathrm{eV}$ that also is taken as a low value.

\section{COUPLER SHAPE OPTIMIZATION}

Fig. 2 shows the geometry of the cavity with a twincoaxial coupler and some details of the coupler. Because the ERL injector cavity coupler has to transfer an average power of $100 \mathrm{~kW}$, we have decided to use a $60 \mathrm{~mm}$ diameter outer conductor [4]. To minimize losses in the inner conductor we chose an impedance of $60 \mathrm{Ohm}$. A shape of the antenna tips presents a bended elliptic disc. Parameters of this disc were found from two requirements: to keep the dipole mode frequency as high as possible, and provide necessary coupling with the cavity. The bending of the disc, for example, increases coupling by $20 \%$ as compared with a flat disc.
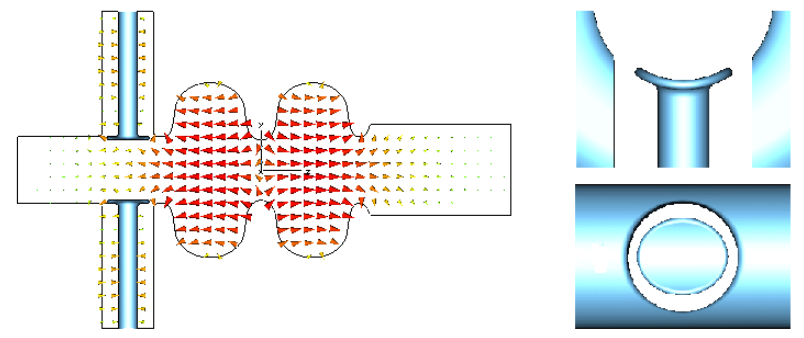

Fig. 2. Geometry of the cavity with the fundamental mode excited in it and details of the coupler.

The coupling is adjustable by changing the depth of penetration of the antenna tip (Fig. 3). The edges of the cross-sections between the outer tube of the coaxial line and the beam pipe are rounded with a radius of $4 \mathrm{~mm}$. This helps to exclude high values of electric field on the surface and increases coupling, but decreases the dipole mode frequency. Finally (see Fig. 2), the smaller radius of the elliptic cross-section and the ratio of its half-axes were used for optimization.

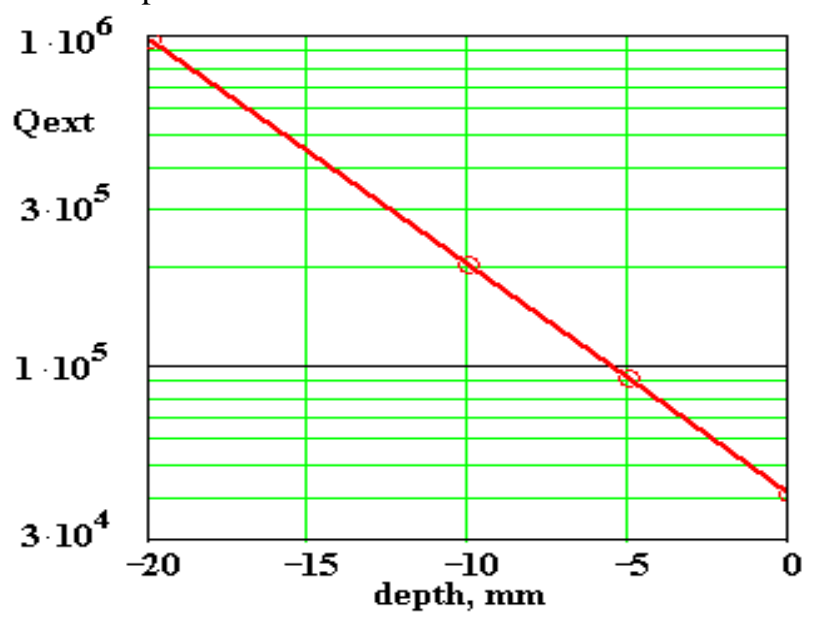

Fig. 3. Dependence of $Q_{\text {ext }}$ on the depth of antenna penetration measured relative to the inner cavity iris. 
The final value $Q_{\mathrm{ext}}=4.08 \times 10^{4}$ satisfies the specified requirements. The value of the $R / Q$ obtained with MWS is $218 \mathrm{Ohm}$.

The value of the transverse kick is recalculated in comparison with the previous estimation [4], because the position of the coupler had changed from the large beam pipe to the narrow one, and geometry of the antenna tip had changed as well. For geometric asymmetry $\Delta l$ in penetration of the antenna tip when the penetration is maximal, the kick is

$$
\left(\Delta p_{t} / \Delta p_{l}\right)_{\text {geo }}=(-0.2-2.2 i) \cdot 10^{-4} \Delta l / \mathrm{mm},
$$

where $\Delta p_{t}$ is the change of the transverse momentum, and $\Delta p_{l}$ is the change of the longitudinal momentum of particles after passing the cavity along its axis. For phase asymmetry $\Delta \varphi$ between two antennas the kick is

$$
\left(\Delta p_{t} / \Delta p_{l}\right)_{\text {phase }}=(1.1-0.7 i) \cdot 10^{-4} \Delta \varphi / \text { degree }
$$

The kick now is about 3 times bigger than in the previous calculation but still quite tolerable.

\section{PROPERTIES OF THE DIPOLE MODE}

We maintained the condition

$$
\Delta f(\mathrm{TE} 11)=f(\mathrm{TE} 11)-f_{\text {cut-off }} \geq 10 \mathrm{MHz}
$$

for the dipole mode by optimizing the cavity shape to secure adequate propagation. Calculation with both SLANS2 and MWS code gave $\Delta f(\mathrm{TE} 1)=10.0 \mathrm{MHz}$ for the final shape.

When two coaxial couplers are added to the geometry, degeneracy of the dipole mode is cancelled, and the mode splits into 2 modes: parallel and transverse mode (with the electric field in the cavity parallel or perpendicular to the coaxial line). Their frequencies changed from the original one but still stayed high enough over the cut-off frequency. These frequencies depend on the coupler geometry, and on the boundary condition at the end of the larger beam pipe. The frequency of the parallel mode depends also on the boundary condition at the ends of the coaxial line. This means that the parallel mode is excited not only in the larger beam pipe but in the coaxial line also and thus has all the more low external $Q$. With the method described in detail in [4] and extended for circular waveguides, the value of $Q_{\text {ext }}$ of the parallel dipole mode was found as $Q_{\mathrm{ext}, \mathrm{p}}=250$. The transverse dipole mode has lower frequency than the initial value (without input coupler) but still propagates into the beam pipe and has $Q_{\text {ext, t }} \sim 1000$.

\section{CONCLUSIONS}

Optimization of the superconducting 2-cell cavitycoupler system for the ERL injector was performed using the MWS 4.0 and SLANS software packages to produce dipole-mode-free and kick-free cavity. Main parameters of the cavity are presented in Table. The completion of calculations allows us to begin production of the injector cavity with the twin-coaxial coupler.
Table. Calculated parameters of the 2-cell cavity with the twin-coaxial coupler.

\begin{tabular}{|c|c|}
\hline Fundamental $\pi$-mode frequency & $1300 \mathrm{MHz}$ \\
\hline$E_{\mathrm{pk}} / E_{\mathrm{acc}}$ & 1.94 \\
\hline$H_{\mathrm{pk}} / E_{\mathrm{acc}}$ & $42.8 \mathrm{Oe} /(\mathrm{MV} / \mathrm{m})$ \\
\hline Coupling cell to cell & $0.7 \%$ \\
\hline$R / Q$, fundamental mode $(\mathrm{FM})$ & $218 \mathrm{Ohm}$ \\
\hline$Q_{\text {ext }}, \mathrm{FM}$, required range & $4.6 \times 10^{4} \ldots 4.1 \times 10^{5}$ \\
\hline $\begin{array}{c}Q_{\text {ext }}, \mathrm{FM}, \text { penetration } \text { depth } \text { of } \\
\text { the antenna }=0 \ldots-15 \mathrm{~mm}\end{array}$ & $4.1 \times 10^{4} \ldots 4.6 \times 10^{5}$ \\
\hline$Q_{\text {ext, }}$, dipole mode, parallel & 250 \\
\hline$Q_{\text {ext, }}$, dipole mode, transverse & 1000 \\
\hline
\end{tabular}

\section{REFERENCES}

[1] Study for a proposed Phase I Energy Recovery Linac (ERL) Synchrotron Light Source at Cornell University, ed. by S. Gruner and M. Tigner, CHESS Technical Memo 01-003 and JLAB-ACT-01-04 (July 4, 2001).

[2] I. Bazarov, et al., "Phase I Energy Recovery Linac at Cornell University", Proceedings of the 8th European Particle Accelerator Conference, Paris, France, June 2002, pp. 644-646.

[3] S. Belomestnykh, et al., "High Average Power Fundamental Input Couplers for the Cornell University ERL: Requirements, Design Challenges and First Ideas," Cornell LEPP Report ERL 02-8 (September 9, 2002).

[4] V. Shemelin, S. Belomestnykh and H. Padamsee, "Low-kick Twin-coaxial and Waveguide-coaxial Couplers for ERL", Cornell LEPP Report SRF 021028-08 (November 28, 2002).

[5] H. Padamsee et al., "Accelerating Cavity Development for the Cornell B-Factory, CESR-B", Proceedings of the PAC'91, pp. 786-788.

[6] S. Mitsunobu et al., "Superconducting RF Activities at KEK", Proceedings of the $5_{\text {th }}$ Workshop on RF Superconductivity, Hamburg, Germany, 1991, pp. 84-94.

[7] D. G. Myakishev, V. P. Yakovlev. "The New Possibilities of SuperLANS code for Evaluation of Axisymmetric Cavities", 1995 Particle Accelerator Conference and International Conference on HighEnergy Accelerators, Texas, May 1995, pp. 23482350.

[8] V. Shemelin, H. Padamsee, S. Belomestnykh, "HOMfree 2-cell cavity with strong input coupler for the SC ERL injector", Cornell LEPP Report SRF 030219-01 (February 19, 2003).

[9] B. Aune et al., "Superconducting TESLA Cavities", Phys. Rev. ST Accel. Beams 3092001 (2000).

[10] P. Ylä-Oijala et al. MultiPac 2.1. Rolf Nevanlinna Institute, Helsinki, 2001. 